\title{
Effects of Adjuvant Hydrotherapy on Functional Status and Mental Relaxation in Patients with Knee Osteoarthritis: Preliminary Study
}

\author{
Eun Young Han, MD ${ }^{1}$, Bo Ryun Kim, $\mathrm{MD}^{1}$, Sang Hee Im, $\mathrm{MD}^{2}$, \\ Jun Hwan Choi, MD ${ }^{1}$, Sun Mi Kim, MD ${ }^{1}$ \\ ${ }^{I}$ Department of Rehabilitation Medicine, Jeju National University Hospital, Jeju, \\ ${ }^{2}$ Department of Rehabilitation, Catholic Kwandong University International St. Mary's Hospital, Catholic Kwandong \\ University College of Medicine, Incheon, Korea
}

Background: The aim of this study is to test if adjuvant hydrotherapy with viscosupplement is beneficial on management of pain, stiffness, function, and mental relaxation in knee osteoarthritis (OA).

Methods: Nineteen patients with OA were randomly assigned to hydrotherapy or control groups. All patients received viscosupplement injections once a week for 3 weeks. Hydrotherapy group $(\mathrm{n}=9)$ had a spa containing green tea, 3 days a week for 2 weeks. The control group $(\mathrm{n}=10)$ received only injections. All patients were assessed at baseline and after the third injection. All were assessed for pain (visual analog scale, VAS), pain severity, and functional status (Western Ontario and McMaster Universities osteoarthritis index, WOMAC), emotional status, quality of life (Euro quality of life health-related quality of life inventory five dimension, EQ-5D), and relative spectral power of alpha waves in electroencephalography (EEG).

Results: Both groups reported a statistically significant reduction of pain at the end of treatment and detailed assessment of pain, and function in WOMAC and the relative power of alpha in electroencephalogram showed statistical significant difference only in the hydrotherapy group. However, there were no significant intergroup differences, except for VAS score.

Conclusion: Adjuvant 2-week hydrotherapy to viscosupplement might have a modest role in the management of pain and functional disability and the mental relaxation in patients with knee OA. Larger, randomized controlled trials with intervention of long term period to determine efficacy in treating knee OA are warranted.

Key Words: Osteoarthritis, Green tea extract, Hydrotherapy, Electroencephalography, Viscosupplement

\section{INTRODUCTION}

Osteoarthritis (OA) is the most common form of chronic arthritis ${ }^{1)}$, causing pain, stiffness, edema, and dysfunction of the involved joint. It has pathologic characteristics

- Received: July 5, 2014 > Revised: August 1, 2014

- Accepted: August 27, 2014

Address for correspondence: Bo Ryun Kim, MD, PhD

Department of Rehabilitation Medicine, Jeju National University

Hospital, 15 Aran 13-gil, Jeju 690-767, Korea

Tel: +82-64-717-1671, Fax: +82-64-717-1131

E-mail: brkim08@gmail.com of both focal loss of articular cartilage and marginal and central formation of new bone ${ }^{2)}$. OA is strongly associated with aging, and is a major disability in elderly people in Korea. It also causes various psychological problems, including depression, a sense of alienation, and apathy, reduces quality of life (QoL), and increases the risk of additional morbidity and mortality ${ }^{2,3)}$.

The objectives of the management of knee OA are to relieve pain, maintain or improve mobility, and minimize disability. Although there are several guidelines on the conservative treatment of $\mathrm{OA}$, most are based on the evidence from pharmacological therapy. However, pharmacological treatments based on symptomatic drugs provide 
only short-term pain relief, and their effects are probably too small to be meaningful to patients ${ }^{4}$. Furthermore, many nonsteroidal anti-inflammatory drugs (NSAIDs) are associated with considerable side effects ${ }^{5}$. In addition, intraarticular injection of long acting corticosteroid is indicated for flares of knee pain, especially if accompanied by effusion $^{()}$; however, many practitioners avoid the longterm use of these injections because repeated use may cause cartilage destruction or systemic infection.

On the other hand, hyaluronan is a highly coiled molecule which acts as viscous lubricant, resists compressive forces, and reduces friction between opposing cartilage surfaces $^{7}$. Hyaluronan is associated with a low incidence of local adverse events and is well tolerated, and is therefore recommended for treating less-advanced knee OA of patients ${ }^{7}$. However, patients receiving intra-articular hyaluronic acid (HA) products are known to complain of pain and/or swelling of the injected joint, and systemic hypersensitivity reactions may occur occasionally.

On the other hand, hydrotherapy or spa is one of the most commonly used nonpharmacological approaches for $\mathrm{OA}^{8)}$ and various recently published systematic reviews and meta-analyses on spa therapy for rheumatic diseases have encouraging evidence of the effect of balneotherapy on knee $\mathrm{OA}^{8,9)}$. However, balneotherapy has a regional limitation and cannot be widely utilized in general. Furthermore, despite the long history and popularity of hydrotherapy, the evidence for its benefit in OA is not clear and it is being challenged to prove its worth.

The current study addressed several objectives. First, as a preliminary study, we evaluated the therapeutic effect of hydrotherapy on pain, stiffness, and function in the elderly patients with OA of knee as an adjuvant treatment method after viscosupplementation. Second, we objectively confirmed the beneficial effects of adjuvant hydrotherapy on mental relaxation using electroencephalography (EEG) and might propose hydrotherapy to create a high-value medical product.

\section{MATERIALS AND METHODS}

\section{Subjects}

Twenty two outpatients who visited the Physical Medicine and Rehabilitation Clinic in Jeju National University between October 26, 2011, and February 28, 2012, for knee pain for at least 3 months and were diagnosed as having bilateral knee OA according to the American College of Rheumatology Classification Criteria ${ }^{10)}$ were recruited in this study. All patients had symptomatic knee pain $>30 \mathrm{~mm}$ as measured by visual analog scale (VAS) for at least 3 months prior to inclusion in the study. Radiological staging was carried out using the KellgrenLawrence method ${ }^{11)}$, and patients with a radiological score of I-III were included in the study.

Patients with severe OA (Kellgren score IV), psychiatric disease, impaired cognition (Mini-Mental Status Examination $<24$ ), and severe radiating pain in the upper extremities, or with peripheral neuropathy were excluded from the study. Patients who had undergone thermal treatments, joint lavage, arthroscopy, or treatment with intra-articular HA or steroid during the previous 3 months were also not eligible for inclusion. Other exclusion criteria were as follows: allergic reaction to green tea, abnormal neurologic signs, previous spinal or musculoskeletal fracture or surgery, pregnancy, or serious medical illness including comorbidity of the heart or brain, varices, systemic bleeding disease, and neoplasm. This study protocol was reviewed and approved by the Jeju University of Institutional Review Board, and that written informed consent for this research project was obtained from all participants.

\section{1) Group assignment}

The patients were randomly assigned to a hydrotherapy group ( $\mathrm{n}=11)$ or a control group $(\mathrm{n}=11)$. Simple randomization was done using a computer-generated table of random numbers. But, after first injection, three participants (two in the spa group and one in the control group) refused evaluation at baseline and were withdrawn.

\section{Intervention}

\section{1) Ultrasound-guided intra-articular knee injection of $\mathrm{HA}$}

HA preparations were administered into the patients' knee joints bilaterally while observing the suprapatellar pouch filling under live ultrasound guidance. The patients reported minimal pain during the procedure and complete injection-related pain relief immediately after the 
injection. All patients in both groups received injections once a week for 3 weeks and were allowed to carry out their daily routines during the study period. The use of symptomatic drugs such as analgesics or NSAIDs and physical modalities were discontinued during the intervention period.

\section{2) Hydrotherapy}

The patients in the hydrotherapy group ( $\mathrm{n}=9 ; 2$ males and 7 females; average, 66.89 \pm 7.41 years) immersed their body, up to the xiphoid level, in a whirlpool bath containing $1 \mathrm{~kg}$ of green tea leaves at $34-36^{\circ} \mathrm{C}$ for 30 minutes. The Jesetter whirlpool bath (Hot Spring Spas N.Z Ltd, Auckland, New Zealand) included 14 jets (two sets of one Motor-Massage DX jet, one Jetstream jet, two rotary Hydromassage jets, one directional Hydromassage jets, four Hydro Stream jets, four directional Precision jets). The treatment room ambient temperature was maintained at around 28$30^{\circ} \mathrm{C}$ to prevent patient body heat loss. Hydrotherapy was performed for 30 minutes a day, 3 days a week for a total duration of 2 weeks. The control group ( $\mathrm{n}=10: 2$ males and 8 females; average, $63.40 \pm 7.46$ years) received only viscosupplement injection without any other interventions.

\section{Evaluation}

In this study, a blind researcher for group assignment gave instructions to the patients for the questionnaires and collected the outcome measurements. All participants were assessed before treatment (at baseline) and after treatment (at the end of the third week). All tests were performed within 48 hours after $1^{\text {st }}$ and $3^{\text {rd }}$ injection.

\section{1) Pain severity and functional assessment}

The variables evaluated were as follows: pain severity was evaluated using a VAS ${ }^{12)}$. The VAS for pain consisted of three 100-mm lines, each of which was labeled with 'no pain' at the left-hand end $(0 \mathrm{~mm})$ and 'very severe pain' at the right-hand end $(100 \mathrm{~mm})^{12)}$. Patients were asked to draw a vertical mark on each line, one on the upper line for their current pain, one on the middle line for the pain at their best (least painful) period during the previous week, and one on the lower line for the pain at their worst (most painful) period during the previous week.
The Western Ontario and McMaster Universities osteoarthritis index (WOMAC) is a multidimensional measure of pain, stiffness, and physical functional disability as an indicator of self-reported disability ${ }^{5}$. WOMAC parameters were scored using the Likert scale (0, no pain; 1 , mild; 2 , moderate; 3 , severe; and 4 , very severe pain), which is recommended by the OMERACT (Outcome Measures in Rheumatology Clinical Trials). The sum of the scores was obtained by adding the scores of five parameters of pain (WOMAC total pain score, W-TPS), two parameters of stiffness (WOMAC total stiffness score, W-TSS), and 17 parameters of physical function (WOMAC total physical function score, W-TPFS ${ }^{5,13,14)}$. The total subscore for WTPS and W-TSS ranged from 0 to 20 and from 0 to 8 , respectively. W-TPFS rated the degree of difficulty experienced in performing each of 17 activities in the preceding 48 hours, with the total subscore ranging from 0 to 68. Higher scores indicate greater levels of difficulty ${ }^{5}$.

\section{2) Emotional status and QoL}

Emotional status and QoL evaluation included depressive mood, which was measured using the Korean version of the Beck Depression Inventory (K-BDI) ${ }^{15}$. Anxiety status was measured using the Korean version of the Beck Anxiety Inventory $(\mathrm{K}-\mathrm{BAI})^{16)}$ and QoL was scored using Euro QoL health-related QoL inventory five dimension (EQ-5D). The BDI is a 21-item scale that gathers information on different symptoms of depression ${ }^{15,17}$. Each item on the scale is scored from 0 to 3. It provides information on both the presence and severity of depression; higher scores indicate the presence of more severe depression.

The BAI consists of 21 anxiety symptoms, with respondents being asked to indicate the extent to which they were bothered by each item "during the past week, including today" ${ }^{16,18)}$. Each responses is scored on a $0-3$ scale ranging from "not at all" to "severely", the inventory has a score range of 0 to 63. As a recommended scoring and interpretation system for the BAI, scores of 10-18 points are classified as mild-moderate anxiety, 19-29 points as moderate-severe, and 30-63 points as severe anxiety ${ }^{16,18)}$.

QoL of the patients was assessed using the Korean version of the EuroQoL EQ-5D Index ${ }^{19)}$. The EQ-5D Index is a widely used measurement of general health status. The instrument comprises a questionnaire with five dimensions: mobility (M), self-care (SC), usual activities(UA), pain/ 
discomfort (PD), and anxiety/depression (AD). Each dimension is represented by one question with three severity levels (no problems, some or moderate problems, and extreme problems) ${ }^{19)}$. Scores were transformed by using utility weights derived from the Korean general population $^{19)}$ and ranged from -1 to 1 , with higher scores indicating better overall health status. The formula producing the EQ-5D Index was as presented in Kim et al. ${ }^{19)}$.

\section{3) Electroencephalography}

(1) EEG recordings and experimental conditions ${ }^{20)}$

The participants were seated in a comfortable arm chair in a sound-attenuated, dimly lit EEG room at different day with injection. After having become accustomed to the atmosphere, the participants were checked for $5 \mathrm{mi}-$ nutes resting EEG collection with eyes closed. To avoid any disturbances and interferences, the recording room was shielded with copper. EEG were recorded using silver-silver chloride cup electrodes (Ag-AgCl) attached by collodion, on Fp1 and Fp2 (prefrontal), on F3 and F4 (frontal), on P3 and P4 (parietal), and on T3 and T4 (temporal) scalp regions of the 10-20 international system $^{21)}$. All electrode impedances were below $5 \mathrm{kX}$. The EEG-measurement-device (LEX3208, LAXTHA Inc., Daejeon, Korea) settings were as follows: $256-\mathrm{Hz}$ sampling rate, 12-bit analog-to-digital converter, 0.6- $\mathrm{Hz}$ highpass filter, 46- $\mathrm{Hz}$ low-pass filter, and $60-\mathrm{Hz}$ notch filter. The EEG data were visually inspected for artifacts. Data contaminated with artifact were discarded.

(2) Relative spectral power and topographic mapping ${ }^{22}$ The raw EEG data were used without any prenormalization. The raw data were used as such except that a $60-\mathrm{Hz}$ notch filter was applied to remove possible artifacts related to power line noise. To estimate the relative power of the EEG data we used a 5-second nonoverlapping sliding window approach. In each window, we computed the relative power in the different subbands: delta $(0.1-4 \mathrm{~Hz})$, theta (4-7 Hz), alpha (7-14 Hz), beta (14-30 Hz) and gamma (30 Hz to Nyquist frequency). The relative power can be described as the average of the squared coefficients of the fast Fourier transform in the defined frequency range ${ }^{13)}$. To obtain the topographic maps, the relative power was computed for all the electrodes and subbands.

\section{Statistical analysis}

We analyzed the data using the SPSS ver. 12.0 (SPSS Inc., Chicago, IL, USA). Descriptive statistics such as mean \pm standard deviation were calculated from the data collected. Chi-square tests were used to compare the demographic factors between two groups. In cases where the anticipated frequency was lower than five, a Fisher exact test was applied. We analyzed the changes of the described test items, including the evaluation of VAS, BAI, EQ-5D, and the relative power in the different subbands before and after treatment within the spa and control groups using the Wilcoxon signed rank test. We compared the differences of above test items before and after treatment between the spa and control groups using the Mann-Whitney test. We calculated Spearman correlation coefficients to examine the relationship between independent and dependent variables using the data from baseline measurements. A p-value $<0.05$ was considered statistically significant.

\section{RESULTS}

\section{Baseline characteristics of the patients in the two groups}

The baseline characteristics of the patients are summarized in Table 1. Baseline comparison of the two groups showed no statistically significant differences in age, gender, mean body mass index, number of patients, baseline severity of pain and anxiety status, QoL, and WOMAC scores $(\mathrm{p}>0.05)$. The majority of the OA patients were women. At baseline, the median parameters of BAI and BDI were within normal range.

\section{Changes in parameters after the 3-week intervention}

Both groups reported a statistically significant reduction in VAS pain scale scores at the end of treatment (Table $2)(p<0.05)$. VAS pain scores were reduced more in the hydrotherapy group than in the control group $(\mathrm{p}=0.04)$.

At the end of treatment, detailed assessment of pain (W-TPS), physical function (W-TPFS) and total scores in 
the WOMAC parameters had improved only in the hydrotherapy group. By contrast, in the control group, none of these parameters had changed (Table 2).

At the end of the treatment, the patients' QoL had not changed significantly in either group (Table 2).

The relative power of alpha waves in $\mathrm{P} 4$ decreased in the spa group $(\mathrm{p}=0.02)$ and additionally, the relative power of alpha waves in Fp1, F3, F4, T3, and P3 tended to be reduced in the spa group $(0.05<\mathrm{p}<0.1)$. However, in the control group, the relative power of alpha waves did not change significantly in any EEG region examined (Table 3).

\section{DISCUSSION}

This study evaluated the therapeutic effect of adjuvant hydrotherapy with the HA injection on pain and functional disability and mental relaxation in the patients with knee OA for the first time. For the beneficial effects of intra-articular HA injection, previous research reviewed it as an effective treatment for early to moderate OA of the knee on pain, function and patient global assessment, especially at the 5- to 13 -week post injection period ${ }^{23)}$. And another recent systematic review reported that its effects peak around 6-8 weeks following administration of $1^{\text {st }}$ hyaluronan, but it concluded that this injection only has ordinary effects on mild to moderate knee OA. Indeed, the clinical guidelines issued by the American Academy of Orthopedic Surgeons ${ }^{24)}$ and Korean Knee Society Subcommittee ${ }^{()}$recently don't recommend for or against the use of intra-articular HA for patients with mild to moderate symptomatic OA of the knee.

However, our results revealed the beneficial effects of intra-articular HA on pain reduction of symptomatic knee $\mathrm{OA}$ and also demonstrated that the adjuvant hydrotherapy had modest influence on the reduction of pain,

Table 1. Baseline general characteristics of the hydrotherapy and control groups

\begin{tabular}{lccc}
\hline \hline Characteristic & Hydrotherapy & Control & p-value \\
\hline Number & 9 & 10 & \\
Gender & & & \\
$\quad$ Male:female & $2: 7$ & $2: 8$ & 0.67 \\
Age (yr) & $66.9 \pm 7.4$ & $63.4 \pm 7.5$ & 0.24 \\
K-L grade & & & 0.93 \\
$\quad$ I:II:III & $2: 3: 4$ & $3: 3: 4$ & \\
Body mass index (kg/m $\left.{ }^{2}\right)$ & $24.86 \pm 1.92$ & $24.40 \pm 1.34$ & 0.72 \\
K-BDI & $6.78 \pm 6.59$ & $8.90 \pm 12.31$ & 0.91 \\
K-BAI & $8.89 \pm 7.04$ & $7.90 \pm 7.81$ & 0.55 \\
EQ-5D & $0.78 \pm 0.09$ & $0.82 \pm 0.08$ & 0.31 \\
Visual analogue scale & $40.0 \pm 13.2$ & $33.0 \pm 10.6$ & 0.28 \\
WOMAC (pain) & $8.7 \pm 5.5$ & $10.7 \pm 8.2$ & 0.97 \\
WOMAC (stiffness) & $3.9 \pm 1.5$ & $3.8 \pm 3.7$ & 0.78 \\
WOMAC (function) & $18.1 \pm 12.3$ & $19.8 \pm 15.8$ & 0.84 \\
WOMAC (total) & $31.8 \pm 19.5$ & $34.6 \pm 26.9$ & 0.99
\end{tabular}

Values are presented as mean \pm standard deviation.

K-L grade, Kellgren-Lawrence grade; K-BDI, the Korean version of the Beck Depression Inventory; K-BAI, the Korean version of the Beck Anxiety Inventory; EQ-5D, Euro quality of life health-related quality of life inventory five dimension; WOMAC, The Western Ontario and McMaster Universities osteoarthritis index.

Table 2. Changes in pain, stiffness, function and quality of life scores of the hydrotherapy and control groups before and after intervention

\begin{tabular}{|c|c|c|c|c|c|c|c|}
\hline \multirow[b]{2}{*}{ Variable } & \multicolumn{3}{|c|}{ Hydrotherapy (hydrotherapy + injection) } & \multicolumn{3}{|c|}{ Control (injection only) } & \multirow{2}{*}{$\begin{array}{l}\text { Intergroup } \\
\text { difference }\end{array}$} \\
\hline & Before & After & $\begin{array}{l}\text { Intragroup } \\
\text { difference }\end{array}$ & Before & After & $\begin{array}{l}\text { Intragroup } \\
\text { difference }\end{array}$ & \\
\hline WOMAC (pain) & $8.7 \pm 5.5$ & $6.6 \pm 5.8$ & $0.02^{*}$ & $10.7 \pm 8.2$ & $8.0 \pm 5.0$ & 0.47 & 0.32 \\
\hline WOMAC (stiffness) & $3.9 \pm 1.5$ & $2.6 \pm 2.4$ & 0.07 & $3.8 \pm 3.7$ & $2.9 \pm 1.7$ & 0.44 & 0.55 \\
\hline WOMAC (function) & $18.1 \pm 12.3$ & $14.2 \pm 10.5$ & $0.02^{*}$ & $19.8 \pm 15.8$ & $12.8 \pm 10.5$ & 0.84 & 0.45 \\
\hline WOMAC (total) & $31.8 \pm 19.5$ & $23.4 \pm 16.6$ & $0.008^{* * *}$ & $34.6 \pm 26.9$ & $26.2 \pm 15.4$ & 0.96 & 0.36 \\
\hline VAS $(\mathrm{cm})$ & $40.0 \pm 13.2$ & $25.6 \pm 10.1$ & $0.006^{* * *}$ & $33.0 \pm 10.6$ & $26.0 \pm 9.6$ & $0.02^{*}$ & $0.04^{*}$ \\
\hline EQ-5D & $0.78 \pm 0.09$ & $0.79 \pm 0.09$ & 0.67 & $0.82 \pm 0.08$ & $0.82 \pm 0.11$ & 0.72 & 0.13 \\
\hline
\end{tabular}

Values are presented as mean \pm standard deviation.

WOMAC, The Western Ontario and McMaster Universities osteoarthritis index; VAS, visual analogue scale; EQ-5D, Euro quality of life health-related quality of life inventory five dimension.

" $\mathrm{p}<0.05$. $" \mathrm{p}<0.01$. 
Table 3. Electroencephalography parameters of the hydrotherapy and control groups before and after therapy

\begin{tabular}{|c|c|c|c|c|c|c|}
\hline \multirow{2}{*}{ RA } & \multicolumn{3}{|c|}{ Hydrotherapy } & \multicolumn{3}{|c|}{ Control } \\
\hline & Before & After & $\mathrm{p}$-value & Before & After & $\mathrm{p}$-value \\
\hline Fp1_RA & $0.48 \pm 0.11$ & $0.44 \pm 0.08$ & 0.09 & $0.42 \pm 0.13$ & $0.40 \pm 0.17$ & 0.39 \\
\hline Fp2_RA & $0.49 \pm 0.11$ & $0.44 \pm 0.10$ & 0.17 & $0.40 \pm 0.15$ & $0.39 \pm 0.18$ & 0.65 \\
\hline F3_RA & $0.51 \pm 0.14$ & $0.46 \pm 0.11$ & 0.09 & $0.45 \pm 0.12$ & $0.42 \pm 0.16$ & 0.29 \\
\hline F4_RA & $0.51 \pm 0.14$ & $0.42 \pm 0.12$ & 0.07 & $0.45 \pm 0.13$ & $0.42 \pm 0.17$ & 0.51 \\
\hline T3_RA & $0.48 \pm 0.10$ & $0.44 \pm 0.09$ & 0.05 & $0.40 \pm 0.14$ & $0.41 \pm 0.15$ & 0.72 \\
\hline T4_RA & $0.45 \pm 0.13$ & $0.39 \pm 0.11$ & 0.17 & $0.44 \pm 0.16$ & $0.40 \pm 0.20$ & 0.17 \\
\hline P3_RA & $0.58 \pm 0.10$ & $0.53 \pm 0.08$ & 0.07 & $0.52 \pm 0.15$ & $0.49 \pm 0.19$ & 0.45 \\
\hline P4_RA & $0.57 \pm 0.12$ & $0.51 \pm 0.10$ & $0.02 *$ & $0.53 \pm 0.15$ & $0.50 \pm 0.20$ & 0.45 \\
\hline
\end{tabular}

Values are presented as mean \pm standard deviation.

RA, relative power of alpha wave; Fp, prefrontal; F, frontal; T, temporal; P, parietal. $\mathrm{p}<0.05$.

functional disability and the mental relaxation of the patients with mild to moderate knee OA at 2-3 weeks after administration of $1^{\text {st }}$ hyaluronan.

Several trials have demonstrated the favorable influence of spa therapy in patients with knee OA, with beneficial effects on the painful symptomatology and functional capacities ${ }^{25,26}$. Most of these trials used thermal mineral water (spa water) which definition and classification are based on its chemical composition ${ }^{25}$. The temperature of the natural spring or welled water must be at least $20^{\circ} \mathrm{C}$ and balneotherapy is traditionally given as bi-daily thermal water baths at temperatures of $34^{-}-38^{\circ} \mathrm{C}$ during a spa therapy course that usually lasts 10-15 days $^{27)}$. In addition to thermal and mineral components, other factors such as buoyancy, immersion and resistance also play important roles in balneotherapy.

In our hydrotherapy protocol, immersion and buoyancy in water, which provides more resistance than air, and a hydro-massage jet might relax muscles, increase tendon extensibility, and facilitate blood circulation ${ }^{28)}$. According to the gate theory, pain relief may be due to the pressure and temperature of the water on skin, and the heat stimuli may also influence muscle tone and pain intensity, helping to reduce muscle spasm and to increase the pain threshold ${ }^{14)}$.

Interestingly, after 2-week hydrotherapy, the relative power of alpha waves has decreased in various brain areas (Fp1, F3, F4, T3, P3, and P4) in the hydrotherapy group. The alpha rhythm is thought to filter the processing of irrelevant sensory inputs in the primary sensory cortex and is inversely correlated with spatial attention ${ }^{29)}$.
The patients with chronic pain demonstrate the attentional bias that leads them to attend excessively to the painful area ${ }^{30)}$, resulting in both hypersensitivity in the painful area and hypoesthesia with deficits in tactile perceptual processing ${ }^{31)}$ in other areas, and in the ability to modulate alpha. Moreover, alpha oscillations likely have a very important function in a variety of cognitive processes because inhibitory mechanisms contribute to diverse biological and behavioral phenomena. Kerr et al. ${ }^{32)}$ reported that meditation enhanced top-down regulation of a 7- to 14-Hz (alpha) cortical oscillation. Furthermore, decreased alpha power is associated with relaxation induced by autogenic training ${ }^{33)}$, and is observed after performing the yoga high-frequency breathing technique ${ }^{34)}$ and receiving massage therapy ${ }^{35)}$. Therefore, hydrotherapy using hydromassage might have a slight positive influence on mental relaxation, which induces improvements of the patient's pain and physical function temporarily, but adequate intervention period with 6 to 8 weeks should be required for increasing the pain threshold or acquiring long-term therapeutic effect on mental relaxation.

Unexpectedly, the control group only showed improvement in the VAS pain score. This might be because the peak effect did not occur within the 3 weeks after the first injection, as mentioned above, which previous studies reported that the peak effects are observed 5- to 6-week postinjection. And counter to our expectations, the study patients with mild to moderate OA did not express a depressive mood, anxiety, or low QoL at baseline. This result was contrary to our previous study in chronic myofascial pain syndrome ${ }^{36}$. That might be due to the 
pain severity. Most of the patients in our previous study had more severe pain and their anxiety was mild to moderate in severity, and their QoL was relatively low, but the patients in this study expressed relatively mild pain, and that is why the therapy might not have improved scores on the psychological inventories.

Overall, our results indicate that the intra-articular injection of HA may be considered to be effective in controlling pain in mild to moderate OA when performed soon after HA injection but provide only limited evidence of adjuvant hydrotherapy for managing functional disability and mental relaxation.

On the other hand, our research has several notable findings. It was the first study that aimed to provide objective evidence of mental relaxation induced by hydrotherapy using EEG. Second, we used hydrotherapy which it is also more easily utilized than balneotherapy that is only accessible regionally. In addition, spa therapy is very popular and does not have disastrous adverse effects. As the elderly population has been increasing rapidly, it can be applied easily to elderly OA patients without special equipment.

This study had several limitations. First of all, this was a pilot study including a small number of patients that aimed to evaluate the effect of adjuvant hydrotherapy on knee OA. The sample size of this study limited the interpretation of the findings and the precise psychophysiological mechanisms of adjuvant hydrotherapy, which remain unknown. Therefore, further study should include more than 28 participants. Secondly, although there was no significant intergroup difference in pain score, the pain score of hydrotherapy group was higher than that of control at baseline and it might affect more reduction in pain score of hydrotherapy group.

Thirdly, chronic inflammation is the leading cause of connective tissue remodeling and destruction in OA, we used green tea as a bath salt. The polyphenols in green tea (GTPs) comprise a mixture that offers promising new options for the development of more effective strategies for the prevention of inflammation associated diseases, including $\mathrm{OA}^{37)}$ and a recent study indicates that Epigallocatechin-3-gallate (EGCG), the major and most active component of GTPs, protects human chondrocytes from interleukin-1a-induced inflammatory responses, and supports the potential of EGCG in OA treatment/prevention ${ }^{38)}$. However, we did not compare the sole effects of green tea spa with hydrotherapy and did not perform an analysis of the water composition because of technical problems and because the absorption rate of the diluted components was thought to be very low, use of EGCG as effective bath salts might be considered. Further studies about the adequate concentrations of quantified green tea should be conducted. Fourthly, OA studies have high rates of placebo response when patient expectations are high $^{39)}$ and adjuvant hydrotherapy may cause the improvements that were seen in pain and functional indexes in the hydrotherapy group and not in the control group. Lastly, we did not compare the two groups during the period during which the effects of HA injection reportedly peak, and we did not verify how long the effects of hydrotherapy last. Finally, our patients did not perform water-based aerobic or strengthening exercise ${ }^{40)}$ because we only wanted to know the sole effect of hydrotherapy.

Further well-designed randomized clinical trials with adequate sample size and with long-term interventional period are warranted to evaluate the effectiveness of water-based exercise in $\mathrm{OA}$.

In conclusion, the results of this pilot study indicate that viscosupplement therapy is effective on pain reduction in mild to moderate OA and a 2-week course of adjuvant hydrotherapy may have modest role in the management of pain, disability and mental relaxation in knee OA during the period soon after the injection. In spite of invasive treatment, adjuvant hydrotherapy to viscosupplementation might apply easily to the patients with knee OA, especially to elderly patients with comorbidities. However, further steps must be taken to enforce these results by conducting a larger, randomized controlled study and following study with estimating number of patients required to evaluate the treatment of knee OA combined with water-based exercise.

\section{ACKNOWLEDGEMENTS}

Following are results of a study on the "Human Resource Development Center for Economic Region Leading Industry" project, supported by the Ministry of Education, Science \& Technology and the National Research Foundation of Korea. The authors gratefully acknowledge Dae Soo Ko for the consultation of green tea materials. 


\section{REFERENCES}

1. Ayis S, Dieppe P. The natural history of disability and its determinants in adults with lower limb musculoskeletal pain. J Rheumatol 2009;36:583-91.

2. Jordan KM, Arden NK, Doherty M, Bannwarth B, Bijlsma JW, Dieppe P, et al. EULAR Recommendations 2003: an evidence based approach to the management of knee osteoarthritis: Report of a Task Force of the Standing Committee for International Clinical Studies Including Therapeutic Trials (ESCISIT). Ann Rheum Dis 2003;62:1145-55.

3. Dieppe PA, Lohmander LS. Pathogenesis and management of pain in osteoarthritis. Lancet 2005;365:965-73.

4. Bjordal JM, Ljunggren AE, Klovning A, Slordal L. Non-steroidal anti-inflammatory drugs, including cyclo-oxygenase-2 inhibitors, in osteoarthritic knee pain: meta-analysis of randomised placebo controlled trials. BMJ 2004;329:1317.

5. McGettigan P, Henry D. Cardiovascular risk with non-steroidal anti-inflammatory drugs: systematic review of population-based controlled observational studies. PLoS Med 2011;8:e1001098.

6. Korean Knee Society Subcommittee on Osteoarthritis Guidelines. Guidelines for the Treatment of Osteoarthritis of the Knee. J Korean Knee Soc 2010;22:69-74.

7. Bert JM, Waddell DD. Viscosupplementation with hylan g-f 20 in patients with osteoarthrosis of the knee. Ther Adv Musculoskelet Dis 2010;2:127-32.

8. Verhagen AP, Bierma-Zeinstra SM, Boers M, Cardoso JR, Lambeck J, de Bie RA, et al. Balneotherapy for osteoarthritis. Cochrane Database Syst Rev 2007;(4):CD006864.

9. Falagas ME, Zarkadoulia E, Rafailidis PI. The therapeutic effect of balneotherapy: evaluation of the evidence from randomised controlled trials. Int J Clin Pract 2009;63:1068-84.

10. Altman R, Asch E, Bloch D, Bole G, Borenstein D, Brandt $\mathrm{K}$, et al. Development of criteria for the classification and reporting of osteoarthritis. Classification of osteoarthritis of the knee. Diagnostic and Therapeutic Criteria Committee of the American Rheumatism Association. Arthritis Rheum 1986;29:1039-49.

11. Kellgren JH, Lawrence JS. Radiological assessment of osteo-arthrosis. Ann Rheum Dis 1957;16:494-502.

12. Boonstra AM, Schiphorst Preuper HR, Reneman MF, Posthumus JB, Stewart RE. Reliability and validity of the visual analogue scale for disability in patients with chronic musculoskeletal pain. Int J Rehabil Res 2008;31:165-9.

13. Mormann F, Kreuz T, Rieke C, Andrzejak RG, Kraskov A, David P, et al. On the predictability of epileptic seizures. Clin Neurophysiol 2005;116:569-87.

14. Melzack R, Wall PD. Pain mechanisms: a new theory. Science 1965;150:971-9.
15. Rhee MK, Lee YH, Jung HY, Choi JH, Kim SH, Kim YK, et al. A standardization study of beck depression inventory II-Korean version (K-BDI): validity. Korean J Psychopathol 1995;4:96-104.

16. Yook SP, Kim ZS. A clinical study on the Korean version of beck anxiety inventory: comparative study of patient and non-patient. Korean J Clin Psycholo 1997;16:185-97.

17. Beck AT, Steer RA, Carbin MG. Psychometric properties of the beck depression inventory: twenty-five years of evaluation. Clin Psychol Rev 1988;8:77-100.

18. Creamer M, Foran J, Bell R. The Beck Anxiety Inventory in a non-clinical sample. Behav Res Ther 1995;33:477-85.

19. Kim MH, Cho YS, Uhm WS, Kim S, Bae SC. Cross-cultural adaptation and validation of the Korean version of the EQ-5D in patients with rheumatic diseases. Qual Life Res 2005;14: 1401-6.

20. Lee JS, Yang BH, Lee JH, Choi JH, Choi IG, Kim SB. Detrended fluctuation analysis of resting EEG in depressed outpatients and healthy controls. Clin Neurophysiol 2007; 118:2489-96.

21. Guideline thirteen: guidelines for standard electrode position nomenclature. American Electroencephalographic Society. J Clin Neurophysiol 1994;11:111-3.

22. Direito B, Teixeira C, Ribeiro B, Castelo-Branco M, Sales F, Dourado A. Modeling epileptic brain states using EEG spectral analysis and topographic mapping. J Neurosci Methods 2012;210:220-9.

23. Bellamy N, Campbell J, Robinson V, Gee T, Bourne R, Wells G. Viscosupplementation for the treatment of osteoarthritis of the knee. Cochrane Database Syst Rev 2006;(2): CD005321.

24. Richmond J, Hunter D, Irrgang J, Jones MH, Levy B, Marx $\mathrm{R}$, et al. Treatment of osteoarthritis of the knee (nonarthroplasty). J Am Acad Orthop Surg 2009;17:591-600.

25. Fioravanti A, Giannitti C, Bellisai B, Iacoponi F, Galeazzi M. Efficacy of balneotherapy on pain, function and quality of life in patients with osteoarthritis of the knee. Int J Biometeorol 2012;56:583-90.

26. Forestier R, Desfour H, Tessier JM, Françon A, Foote AM, Genty C, et al. Spa therapy in the treatment of knee osteoarthritis: a large randomised multicentre trial. Ann Rheum Dis 2010;69:660-5.

27. Karagulle M, Karagulle MZ, Karagulle O, Donmez A, Turan M. A 10-day course of SPA therapy is beneficial for people with severe knee osteoarthritis. A 24-week randomised, controlled pilot study. Clin Rheumatol 2007;26:2063-71.

28. Weber BC, Hoppe KM. Physical agent modalities. In: Braddom RL, editor. Physical medicine \& rehabilitation. 4th ed. Philadelphia: Saunders; 2011. p. 449-67.

29. Foxe JJ, Snyder AC. The role of alpha-band brain oscillations as a sensory suppression mechanism during selective attention. 
Front Psychol 2011;2:154.

30. Moseley GL, Sim DF, Henry ML, Souvlis T. Experimental hand pain delays recognition of the contralateral hand: evidence that acute and chronic pain have opposite effects on information processing? Brain Res Cogn Brain Res 2005;25: 188-94.

31. Moriwaki K, Yuge O. Topographical features of cutaneous tactile hypoesthetic and hyperesthetic abnormalities in chronic pain. Pain 1999;81:1-6.

32. Kerr CE, Sacchet MD, Lazar SW, Moore CI, Jones SR. Mindfulness starts with the body: somatosensory attention and top-down modulation of cortical alpha rhythms in mindfulness meditation. Front Hum Neurosci 2013;7:12.

33. Jacobs GD, Lubar JF. Spectral analysis of the central nervous system effects of the relaxation response elicited by autogenic training. Behav Med 1989;15:125-32.

34. Stancak A Jr, Kuna M, Srinivasan, Dostalek C, Vishnudevananda S. Kapalabhati: yogic cleansing exercise. II. EEG top- ography analysis. Homeost Health Dis 1991;33:182-9.

35. Diego MA, Field T, Sanders C, Hernandez-Reif M. Massage therapy of moderate and light pressure and vibrator effects on EEG and heart rate. Int J Neurosci 2004;114:31-44.

36. Im SH, Han EY. Improvement in anxiety and pain after whole body whirlpool hydrotherapy among patients with myofascial pain syndrome. Ann Rehabil Med 2013;37:534-40.

37. Katiyar SK, Raman C. Green tea: a new option for the prevention or control of osteoarthritis. Arthritis Res Ther 2011;13:121.

38. Akhtar N, Haqqi TM. Epigallocatechin-3-gallate suppresses the global interleukin-1beta-induced inflammatory response in human chondrocytes. Arthritis Res Ther 2011;13:R93.

39. Cherkin D. Spa therapy: panacea or placebo? Med Care 1998;36:1303-5.

40. Kim SB, Kwak H. The importance of nonpharmacologic management in osteoarthritis. J Korean Geriatr Soc 2005;9: 169-74. 